\title{
Aclimatacào de peixe de respiraçào aérea facultativa a água hypóxica: afinidade pelo oxigênio do sangue e agentes alostéricos (*)
}

\author{
Roy E. Weber (') \\ Stephen C. Wood ( ${ }^{2}$ ) \\ Bonnie J. Davis ( ${ }^{3}$ )
}

\begin{abstract}
Resumo
Afinidades pelo oxigênio do sangue, concentraçōes eritrocíticas de nucleosídeo trifosfato (NTP) e outros parâmetros hematológicos, foram medidos em peixes de respiração aérea facultativa do Amazonas, depois da aclimatação em água oxigenada ("normóxica") e água hipóxica $\left(\mathrm{PO}_{2}=125\right.$ a $135 \mathrm{e}$ 20 a $25 \mathrm{~mm} \mathrm{Hg}$, respectivamente). No bagre, Hipostomus sp. e Pterygoplichtys sp. a hipóxia leva a interminentes subidas à superfície para tomar ar, resultando em níveis mais baixos de NTP, principalmente pelo significativo decréscimo de guanosina 3-fosfato (GTP). Os subsequientes aumentos na afinidade pelo $\mathrm{O}_{2}$ do sangue aparecem adaptados às tensões de $\mathrm{O}_{2}$ internas médias em tempos mais baixos. Nenhuma troca semelhante foi vista na enguia Synbranchus, a qual respira quase que continuamente ar, quando mantida em água hipóxica. Os resultados, são discutidos em termos de sua significância adaptativa e comparada com os dados sobre a temperatura do peixe.
\end{abstract}

\section{INTRODUÇÃo}

O rio Amazonas contém a maior fauna ictiológica de água doce do mundo. As condições físico-químicas de suas águas variam grandemente, no espaço e no tempo e a fauna ictiológica concordantemente exibe divergentes e múltiplas adaptações à fisiologia respiratória. Particularmente notável é a diversidade de adaptações orgânicas à respiração aérea (cf. Johansen, 1970) que parecem ter evoluído, em resposta às baixas tensões de $\mathrm{O}_{2}$ geralmente encontradas nos brejos e nos igarapés do Amazonas. Isto inclui modificações das guelras a fim de que eles não se fechem no ar (enguia amazônica Synbranchus), o desenvolvimento das papilas bucais (enguia elétrica,
Electrophorus) e a modificação por trocas respiratórias gasosas da bexiga de ar (Erythrinus, Arapaima) ou aparelho gastrointestinal (os bagres Ancistris, Hypostomus e Pterygoplichthys). Muitos destes peixes são de respiração aérea facultativa os quais respiram por brânquias em água oxigenada, mas também respiram ar em água hipóxica. Este trabalho diz respeito às adaptações no sangue, seguindo a troca de respiração aquática para a aérea e vice-versa.

Estudos anteriores mostraram que a afinidade de $\mathrm{O}_{2}$ no sangue do peixe em que falta a habilidade da respiração bimodal, é modulada pelas tensões de $\mathrm{O}_{2}$ ambientais, principalmente através das variações nas concentrações eritrocíticas de nucleosideos trifosfatos (NTP) adenosina trifosfato (ATP) guanosina trifosfato (GTP). (Wood \& Johansen, 1972, 1973a; Wood et al. 1975; Geoghegan \& Poluhowich, 1974; Weber et al., 1975; Kaloustian \& Poluhowich, 1976). Estes agentes alostéricos diminuem a afinidade de $\mathrm{O}_{2}$ da hemoglobina visto que 2,3-difosforoglicerato (DPG) atua nos glóbulos vermelhos dos mamíferos (Benesch \& Benesch, 1967; Chanutin \& Curnish, 1967). Na enguia Anguilla foi notado que as diminuições de NTP dos glóbulos vermelhos sob condições hipóxicas, aumentam a afinidade de $\mathrm{O}_{2}$ sanguíneo não somente pela interação alostérica direta reduzida, mas também, porque afeta a distribuição de Donnan, de prótons, através das membranas dos glóbulos vermelhos. Isto aumenta $\mathrm{opH}$ intraeritrocítico das enguias em ambientes hipóxicos, e assim, a afinidade de $\mathrm{O}_{2}$ da hemoglobina via o efeito Bohr (Wood \& Johansen, 1973). Além disso, os efeitos dire-

$\left({ }^{\circ}\right)$ - Versão original Inglesa publicada em Comp. Blochem. Physiol. vol. 62A (1). 1979.

(1) - Department of Zoophysiology, University of Aarhus, DK-8000 Aarhus C.. Denmark.

(2) - Department of Physiology, University of New Mexico, Albuquerque, New Mexico 87131, U.S.A.

( 3 ) - Department of Biology, San Francisco State University, San Francisco, California 94132, U.S.A. 
tos de NTP parecem ser atribuíveis à alta sensibilidade do componente catódico da hemoglobina ao GTP. (Weber et al., 1976) .

Nossa participação na expedição do barco laboratório "Alpha Helix no rio Amazonas, próximo de Manaus, Brasil, deu-nos oportunidade para investigar a ocorrência de respostas adaptacionais correspondentes, no sangue de peixes com respiração aérea facultativa. Esta investigação diz respeito à adaptação à água hipóxica dos bagres Hypostomus sp. e Pterygoplichthys sp. nos quais o estômago foi modificado para servir nas trocas gasosas entre sangue $\mathrm{e} o$ ar ritmicamente respirado (Carter, 1935) e a enguia Amazônica, Synbranchus marmoratus. Estudos paralelos sobre os bagres investigaram as mudanças no balanço ácidobase e sangue-tampão associado com aclimatação hipóxida (Wood et. al., 1978) e os efeitos dos fatores orgânicos maiores dos glóbulos vermelhos, sobre a ligação de $\mathrm{O}_{2}$ das hemoglobinas fracionadas e compostas. (Weber \& Wood, 1978).

\section{MATERIAL E MÉTODOS}

Os espécimes do bagre Hypostomus sp. (") Pterygoplichthys sp. (membros da ímília Loricariidae, que são extremamente distinguíveis pelo número de raios dorsais, 9 e 13 respectivamente) e da enguia da Amazônia Synbranchus marmoratus (Synbranchidae) foram obtidos do rio Solimões, cerca de $150 \mathrm{~km}$ a oeste de Manaus, Brasil. Todos os espécimes da Pterygoplichthys usados, pertencem à "espécie I" (cf. Fink, 1977). Os bagres variaram de 50 a $115 \mathrm{~g}$ de peso e as enguias de 160 a $550 \mathrm{~g}$. Exemplares de cada uma das espécies foram aclimatados, ou em água oxigenada ("normóxica") ou em água hipóxica por 4-7 dias, a cerca de $30^{\circ} \mathrm{C}$ nos aquários $(90 \times 50 \mathrm{~cm})$ contendo $25 \mathrm{~cm}$ de água aproximadamente. As tensões de $\mathrm{O}_{2}$ nos tanques normóxicos variaram entre 125 e $135 \mathrm{~mm}$ de $\mathrm{Hg}$. Nos tanques hipóxicos, os peixes por si mesmos reduziram a tensão $\mathrm{O}_{2}$ para um nível relativamente estável entre 20 e $25 \mathrm{~mm}$ de $\mathrm{Hg}$. Ambos os grupos tinham acesso normal ao ar normal. Em água bem arejâda, nenhuma respiração aérea foi observada. No meio ambiente hipóxico, no en- tanto, o bagre foi observado regularmente na superfície engolindo ar (aproximadamente cada 5 minutos). Em água hipóxica, o Synbranchus conservou seu focinho mais ou menos permanentemente acima da superfície da água.

Amostra de sangue foram tiradas através de punção cardíaca, utilizando-se seringas heparinizadas. Valores de hematócitos foram determinados pela centrifugaçắo do sangue em tubos capilares. Tensões de $\mathrm{O}_{2}$ puro ou com $\mathrm{N}_{2}$ puro e misturados anaerobicamente em proporções fixadas de sangue completamente oxigenada e desoxigenada em tubos capilares de $125 \mu$ l para produzir níveis conhecidos de saturação de $\mathrm{O}_{2}$. Um radiômetro BMS-2 Mk II foi usado para equilibrar as amostras de sangue, e os valores de $\mathrm{PO}_{2}$ e $\mathrm{pH}$ foram medidos com eletrodos do Radiômetro, usando-se um BMS-3. Para determinar o efeito Bohr quantidades variáveis de $\mathrm{CO}_{2}$ foram misturadas com os gases equilibrantes, usando-se bomba misturadora de gás Wösthoff.

As concentrações de hemoglobina foram determinadas espectrofotomicamente (espectrofotômetro Bechman DU), usando-se os coeficientes de extinção $\propto$ e $\beta$ para oxi-hemoglobina humana (Antonini \& Brunori, 1971). Para as dosagens de concentração no sangue total, amostras de $20 \mu \mathrm{l}$ foram rapidamente misturadas com alíquotas de $5 \mathrm{ml}$ de água destilada. Esperimentos de controle mostraram uma boa concordância entre estes valores e aqueles obtidos por conversão ao derivado de cianometemoglobina (reagentes Clay Adams Co.). Os níveis totais de nucleosídeo trifosfato (NTP) no sangue, foram determinados usando-se o teste químico da Sigma. As contribuições de ATP e GTP foram estimadas pela cromatografia de camada como previamente descrita (Weber et. al., 1976). Os níveis de fosfato celular foram calculados a partir dos níveis de hematócritos, presumindo-se que todos os fosfatos orgânicos são intracelulares.

\section{RESULTADOS E INTERPRETAÇÃo COMPARATIVA}

A afinidade pelo oxigênio do sangue de Hypostomus, aclimatado à água hipóxica são significativamente maiores do que nos espécimes conservados em água oxigenada (normóxica") (Fig. 1). Em pH 7,4 e a $30^{\circ} \mathrm{C}$, os valo-

( $)$ - Hypostomus é referido freqüentemente como Plecostomus. 
res de $\mathrm{P}_{50}$ estão próximos de 15 e $25 \mathrm{~mm} \mathrm{Hg}$, respectivamente. Também na hipóxia resultou um efeito Bohr significativamente mais baixo $\left(\Delta \log \mathrm{P}_{50} / \Delta \mathrm{pH}=-0,18\right.$ vs. $-0,32$ para peixes normóxicos).

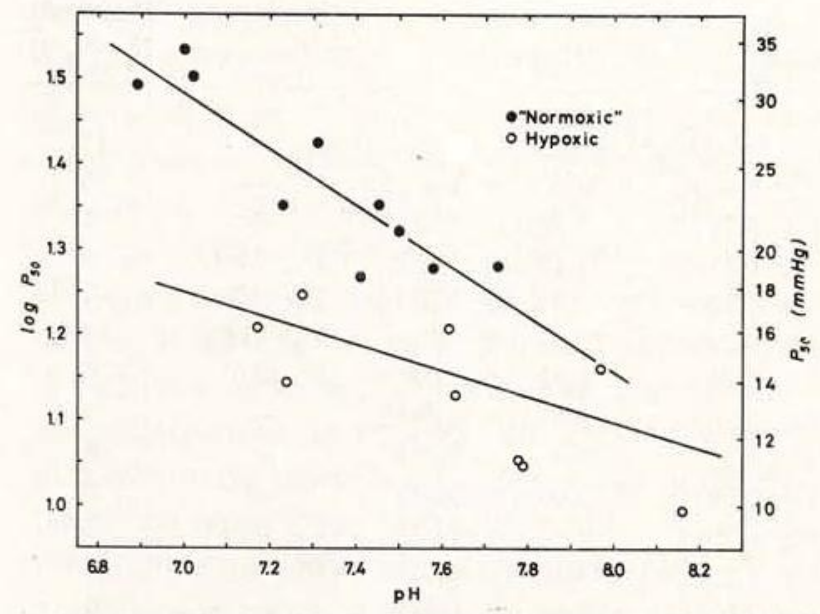

Fig. 1 - Tensão de semi-saturação de oxigênio $\left(\mathrm{P}_{50}\right)$ e sua dependência de $\mathrm{pH}$ do sangue de Hypostomus sp. aclimatados à água hipóxica $\left(0, \mathrm{PO}_{2}=20\right.$ $-25 \mathrm{~mm} \mathrm{Hg})$ e água "normóxica" $\left(0, \mathrm{PO}_{2}=125-\right.$ $135 \mathrm{~mm} \mathrm{Hg}$ ). As equações de regressão para espécies hipóxicas e "normóxicas" são respectivamente: $\log \mathrm{P}_{50}=2.5-0.18 \mathrm{pH}(\mathrm{N}=9, \mathrm{r}=-0.72)$ espécimes hipóxicas), e $\log \mathrm{P}_{50}=3.7-0.32 \mathrm{pH}(\mathrm{N}=10$, $r=-0.89$ ) (espécimes "normóxicas).

A afinidade pelo $\mathrm{O}_{2}$ mais elevada nos peixes hipóxicos correlaciona-se com concentrações mais baixas de NTP (Tabela I, Fig. 2) . A concentração inferior de fosfato em peixe hipóxico é também compatível com o efeito Bohr mais baixo nestes animais, em vista das ligações mais fortes dos anions fosfato, com a diminuição do $\mathrm{pH}$. Separadamente destas interações alostéricas diretas, é provável que o nível inferior de NTP em eritrócitos de peixes hipóxicos aumentará posteriormente a afinidade de $\mathrm{O}_{2}$ na hemoglobina, pelo seu efeito no $\mathrm{pH}$ intracelular (Duhm, 1971; Wood \& Johansen, 1973a). A aclimatação hipóxica induziu a uma diminuição similar nos níveis eritrocíticos de NTP em Pterygoplichthys (Tabela I, Fig. 2) e B. J. Davis (resultados inéditos) tinha observado a mesma resposta em Ancistris chagresi, uma espécie do Panamá.

A separação cromatográfica de NTP revelou que em ambos os bagres, o sangue de indivíduos normóxicos contém um pouco mais
GTP do que a ATP. Entretanto, considerando que o ATP diminuiu apenas ligeiramente durante a aclimatação hipóxica, os niveis de GTP em Hypostomus e Pterygoplichthys diminuiram significativamente $(P<0,001$ e $P<0,01$, respectivamente). Estas descobertas (Tabela I, Fig. 2) sugerem que as trocas observadas na afinidade pelo $\mathrm{O}_{2}$ do sangue são primariamente devidas às variações nos níveis eritrocíticos do GTP.

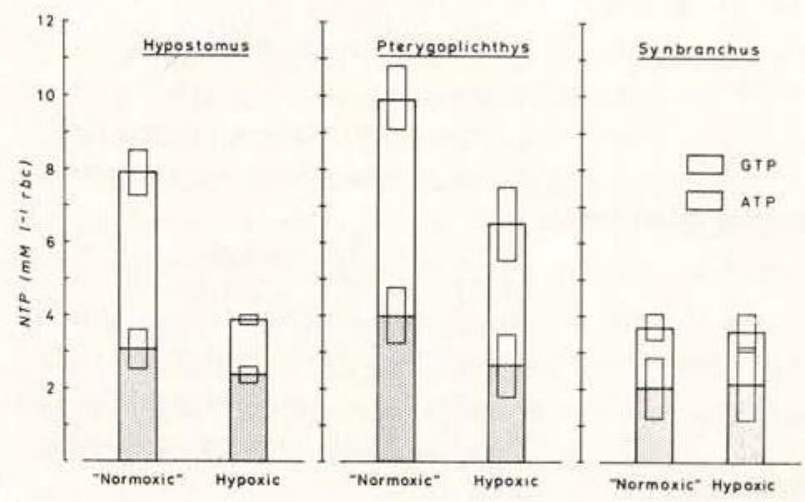

Fig. 2 - Concentrações em mM por litros de globulos vermelhos de ATP (escurecido) e GTP (branco) em Hypostomus, Pterygoplichthys, e Synbranchus aclimatados a água hipóxica ou "normóxica"

Eritrócitos de Synbranchus continham um pouco mais de ATP do que GTP (cerca de 2,0 e $1,5 \mathrm{mMx}^{-1}$ nos glóbulos vermelhos, respectivamente) (Tabela I). Em contraste aos resultados nos glóbulos vermelhos do bagre, nenhuma troca significativa no nível de qualquer fosfato foi observado em condições de aclimatação hipóxica.

Em nenhuma das espécies estudadas a aclimatação hipóxica afetou significativamente o hematócrito. Os níveis de hemoglobina no sangue do bagre, indicaram as capacidades de $\mathrm{O}_{2}$ de cerca de 11 e 9 vol. $\%$ aclimatados "hipóxicas" e "normóxicos", respectivamente; em Synbranchus os valores correspondentes foram de $22 \mathrm{vol} \%$ (Tabela I). Estes peixes então diferem da enguia européia Anguilla onde um nível aumentado de hemoglobina sob condição hipóxica aumenta a utilização do $\mathrm{O}_{2}$ ligado à hemoglobina, resultando em um rendimento cardíaco menor para uma dada liberação de $\mathrm{O}_{2}$ aos tecidos (Wood \& Johansen, 1973b). 
TABELA I - As afinidades de oxigênio e características hematológicas do sangue de peixe com respiração aérea facultativa aclimatadas a condições "normóxicas" $\left(\mathrm{PO}_{2}=125\right.$ a $\left.135 \mathrm{~mm} \mathrm{Hg}\right)$ e hipóxicas $\left(\mathrm{PO}_{2}=20\right.$ a $25 \mathrm{~mm} \mathrm{Hg}$ ). (As capacidades de oxigênio do sangue estão calculadas pela concentração de hemoglobina).

\begin{tabular}{|c|c|c|c|c|c|c|}
\hline Espécie & & "Normóx & xica" & "Hурб́хіса" & $\begin{array}{c}\text { Diferenças } \\
\text { Significativas }\end{array}$ & $\begin{array}{l}\text { Graus de } \\
\text { liberdade } \\
\left(\mathrm{N}+\mathrm{N}_{2}-2\right)\end{array}$ \\
\hline \multirow[t]{8}{*}{ Hypostomus } & afinidade de oxigênio & $\log P_{50}$ & $=3,7-0,32 \mathrm{pH}$ & $\log P_{50}=2,5-c$ & $0,18 \mathrm{pH}-$ & 17 \\
\hline & Het & 31,5 & $( \pm 6,0)$ & $31,1( \pm 1,4)$ & - & 6 \\
\hline & [ATP] (mM/1 célula) & 3,07 & $( \pm 0,54)$ & $2,38( \pm 0,23)$ & - & 4 \\
\hline & [GTP] (mM/1 célula) & 4,84 & $( \pm 0,61)$ & $1,49( \pm 0,11)$ & $\mathrm{P}<0,001$ & 4 \\
\hline & [ATP+GTP] (mM/1 célula) & 7,91 & $( \pm 1,10)$ & $3,87( \pm 0,21)$ & $\mathrm{P}<0,01$ & 4 \\
\hline & [Heme] (mM/1 célula) & 15,59 & $( \pm 0,22)$ & $12,63( \pm 0,64)$ & $\mathrm{P}<0,001$ & 6 \\
\hline & {$[\mathrm{ATP}+\mathrm{GTP}] /[$ heme $](\mathrm{M} / \mathrm{M})$} & 0,47 & $( \pm 0,09)$ & $0,28( \pm 0,04)$ & $P<0,01$ & 6 \\
\hline & $\mathrm{O}_{2}$ capacidade (vol. $\%$, sangue) & 11,15 & $( \pm 2,36)$ & $8,81( \pm 0,64)$ & - & 6 \\
\hline \multirow[t]{7}{*}{ Pterygoplichthys } & Het & 28,7 & $( \pm 6,1)$ & $23,4 \quad( \pm 2,9)$ & - & 6 \\
\hline & [ATP] (mM/1 célula) & 3,99 & $( \pm 0,76)$ & $3,66( \pm 0,66)$ & - & 6 \\
\hline & [GTP] (mM/1 célula) & 5,90 & $( \pm 0,92)$ & $2,88( \pm 1,00)$ & $(\mathrm{P}<0,01)$ & 6 \\
\hline & [ATP+GTP] (mM/1 célula) & 9,89 & $( \pm 1,63)$ & $6,54( \pm 1,83)$ & $(P<0,05)$ & 6 \\
\hline & [Heme] (mM/1 célula) & 17,40 & $( \pm 2,02)$ & $16,48( \pm 1,89)$ & - & 6 \\
\hline & {$[\mathrm{ATP}+\mathrm{GTP}] /[$ heme $](\mathrm{M} / \mathrm{M})$} & 0,57 & $( \pm 0,10)$ & $0,40( \pm 0,12)$ & - & 6 \\
\hline & $\mathrm{O}_{2}$ capacidade (vol. $\%$, sangue) & 11,05 & $( \pm 1,89)$ & $8,64( \pm 1,60)$ & - & 6 \\
\hline \multirow[t]{7}{*}{ Synbranchus } & Het & 48,8 & $( \pm 5,4)$ & $53,5 \quad( \pm 8,1)$ & - & 5 \\
\hline & [ATP] (mM/1 célula) & 2,03 & $( \pm 0,86)$ & $2,16( \pm 1,03)$ & - & 5 \\
\hline & [GTP] (mM/1 célula) & 1,67 & $( \pm 0,37)$ & $1,37( \pm 0,57)$ & - & 5 \\
\hline & [ATP+GTP] (mM/1 célula) & 3,70 & $( \pm 1,21)$ & $3,54( \pm 0,61)$ & - & 5 \\
\hline & [Heme] (mM/1 célula) & 20,59 & $( \pm 0,69)$ & $17,63( \pm 2,44)$ & - & 5 \\
\hline & $\mathrm{ATP}+\mathrm{GTP} /[$ heme $](\mathrm{M} / \mathrm{M})$ & 0,18 & $( \pm 0,06)$ & $0,20( \pm 0,05)$ & 一 & 5 \\
\hline & $\mathrm{O}_{2}$ capacidarle (vol. $\%$, sangue) & 22,49 & $( \pm 2,68)$ & $21,08( \pm 4,23)$ & - & 5 \\
\hline
\end{tabular}

\section{DISCUSSÃo}

As afinidades mais elevadas pelo $\mathrm{O}_{2}$ no sangue e as mais baixas concentrações de cofatores eritrociticos alostéricos nos peixes hipóxicos, foram inesperados na base da suposıção de que durante a respiração aérea as tensōes de oxigênio sanguíneo serão pelo menos tão altas quanto as tensões quando o peixe respira água bem oxigenada. As respostas às propriedades respiratórias do sangue do bagre . entretanto, relembram aquelas previamente registradas em peixes das regiões temperadas, nos quais falta a habilidade de respiração bimodal, onde afinidade aumentada de $\mathrm{O}_{2}$ em peixes hipóxicos melhora a carga do $\mathrm{O}_{2}$ do sangue nas guelras e a utilização de $\mathrm{O}_{2}$ pelos tecidos. Os resultados indicam que, apesar da respiração aérea, as tensões internas de $\mathrm{O}_{2} \mathrm{em}$ bagres, expostos a águas hipóxicas diminuiram a niveis que são bastante baixos para induzir depressão de fosfato eritrocítico e que o au- mento seguinte da afinidade pelo $\mathrm{O}_{2}$ no sangue é uma resposta às tensões de $\mathrm{O}_{2}$ internas médias em vez das tensões de $\mathrm{O}_{2}$ no médio respiratório (ar).

Há diversas maneiras pelas quais os aumentos das afinidades pelo $\mathrm{O}_{2}$ no sanguc podem ser adaptativos ao transporte eficiente de $\mathrm{O}_{2}$ durante a respiração aérea. Primeiramente ele favorecerá a maior depressão de $\mathrm{O}_{2}$ do ar respirado pelo peixe, seguindo intervalos maiores de tempo entre as emersões para a superfície da água. Em segundo lugar, poderia ser também importante em vista da evidência da troca gasosa menos eficiente durante a respiração aérea, resultando em tensões inferiores de $\mathrm{O}_{2}$ do sangue (Carter, 1931). Nas guelras, a vascularização é mais densa, o sangue está em contato mais restrito com a superfície respiratória, e a área respiratória geralmente excede esta no intestino. Carter (1931) estimou que no Hoplosternum a super- 
fície intestinal respiratória é aproximadamente a metade desta nas guelras da maioria dos outros peixes.

A pressão seletiva favorecendo a alta afinidade pelo $\mathrm{O}_{2}$ do sangue pode também depender das adaptações circulatórias comuns nos peixes de respiração aérea. Isto se refere ao desvio respiratório do sangue referente no sistema venoso, que resulta na perfusăo arterial sistêmica com sangue venoso misturado, (Carter 1935; Johansen 1970) pelo qual uma afinidade pelo $\mathrm{O}_{2}$ aumentada melhoraria a perda de eficiência causada pela condição mista do sangue arterial. Semelhante significância adaptativa está de acordo com a relativamente alta afinidade pelo $\mathrm{O}_{2}$ do sangue da enguia elétrica, Electrophorus (Johansen et. al. 1968). Finalmente, a afinidade aumentada pelo $\mathrm{O}_{2}$ do sangue em bagre durante a respiração aérea, deveria diminuir a perda de $\mathrm{O}_{2}$ nas águas hipóxicas em redor.

Nas espécies de bagres estudadas, as trocas quantitativas nos cofatores de fosfato eritrocítico indicam que o GTP desempenhará um papel mais importante do que o ATP na modulação da afinidade pelo oxigênio sanguíneo. Isto está de acordo com as observações sobre outros peixes, que contenham significativas concentrações eritrocíticas de GTP tais como a enguia Anguilla ou o peixe pulmenado Protopterus (Weber et al., 1975, 1976; Johansen et al., 1976). Tentou-se especular qual é o papel do GTP nestas espécies isentas de ATP envolvido no metabolismo energético dos eritrócitos de peixes metabolicamente ativos. Correlacionando-se o maior efeito de GTP do que ATP sobre a afinidade pelo oxigênio da hemoglobina do peixe (Lykkeboe et al., 1975; Weber et al., 1975; Kaloustian \& Poluhowich 1976; Weber \& Wood, 1978) está o descobrimento (Weber, inédito) de que a influência modulante do GTP sobre a afinidade de $\mathrm{O}_{2}$ é menos inibida do que a de ATP nas mesmas concentrações, pela complexidade com cátions bivalentes.

Um problema associado com a adoção do hábito de respiração aérea é uma retenção elevåda de $\mathrm{CO}_{2}$ (Howell, 1970), o qual poderia diminuir o $\mathrm{pH}$ do sangue e assim a afinidade do $\mathrm{O}_{2}$ afetando o resultado dos níveis diminuí- dos de cofator nos espécimes aclimatados a hipóxia, conforme foi mostrado em um trabalho desta coletânea (Wood et al., 1378) isto é prevenido no bagre pelo aumento de bicarbonato no plasma, o qual estabiliza o pH do sangue durante a respiração aérea.

A ausência de respostas hipóxicas similares na afinidade de $\mathrm{O}_{2}$ e nas concentraçōes do cofator no sangue de Synbranchus é consistente com seu comportamento respiratório diferente, e com a observação (Johansen, 1966) de que as saturações arteriais de $\mathrm{O}_{2}$ durante a respiração aérea excedem daquelas durante a respiração aquática que mostram a alta eficiência das guelras nas trocas gasosas aéreas. A ausência de diminuições adaptativas nos níveis eritrocíticos de NTP nesta espécie reforça assim a interpretação de que os níveis diminuidos de fosfato dos glóbulos vermelhos no bagre hipóxico são adaptadas ao transporte interno de $\mathrm{O}_{2}$ a baixas tensões de $\mathrm{O}_{2}$.

\section{AGRADECIMENTOS}

Esta pesquisa foi feita a bordo do barco "Alpha Helix" na expedição ao Amazonas em 1976 e foi financiada pela bolsa PCM-75-06451 da Nation Science Foundation (NSF), da bolsa HL-18026 (S.W.) dos National Institutes of Health e da bolsa 511-6734 (R.E.W.) do Danish Natural Science Research Council. Agradecimentos são também devidos ao governo brasileiro por permitir a expedição.

\section{SUMMary}

Blood oxygen affinities, erythrocytic nucleoside triphosphate concentrations (NTP) and other hematological parameters were measured in facultative air-breathing fish from the Amazon after acclimation to well aerated ("normoxic") and hypoxic water $\left(\mathrm{PO}_{2}=125\right.$ to 135 and 20 to $25 \mathrm{~mm}$, respectively). In the armored catfish Hypostomus sp. and Pterygoplichthys sp., hypoxia induces intermittent surfacing to gulp air and results in lower NTP levels, chiefly through significant decreases in guanosine triphosphate (GTP). The subsequent increases in blood $\mathrm{O}_{2}$ affinity appear adaptive to lowered time average internal $\mathrm{O}_{2}$ tensions. No similar changes were seen in the eel Synbranchus which breathes air almost continuously when kept in hypoxic water. The results are discussed in terms of their adaptive significance, and compared with data on temperate fish. 


\section{BIBLIOGRAFIA}

ANTONINI, E. \& BRUNORI, M.

1971 - Hemoglobin and myoglobin in their reaction with ligands. Amsterdam, North Holland Publishing, p.p. 13-39.

BENESCH, R. \& BENESCH, R.E.

1967 - The effect of organic phosphates from the human erythrocyte on the allosteric properties of hemoglobin. Biochem. Biophys. Res. Commun., 26:162-167.

CARTER, G.S.

1931 - Aquatic and aerial respiration in animals. Biol. Rev., 6:1-35.

1935 - Reports of the Cambridge expedition to British Guiana, 1933. Respiratory adaptations of the fishes of the forest waters, with descriptions of the accessory respiratory organs of Electrophorus electricus. (Linn.) (= Gynotus electricus auctt,) and Plecostomus plecostomus (Linn.). J. Linn. Soc. London, 39:219-233.

Chunutun, A. \& CURnish, R.

1967 - Effect of organic and inorganic phosphates on the oxygen equilibrium of human hemoglobin. Arch. Biochem. Biophys., 121:96-101

DUHм, J.

1971 - Effects of 2,3 diphosphoglycerate and other organic phosphate compounds on oxygen affinity and intracellular $\mathrm{pH}$ of human erythrocytes. Pflügers Arch., 326:341-356

EDWARDS, M.J. \& MARTIN, R.T,

1967 - Mixing technique for the oxygen-hemoglobin equilibrium and Bohr effect. $J$. Appl. Physiol., 21:1898-1902 .

Geoghegan, W.D. \& Poluhowich, J.J.

1974 - The major organic phosphates of the American eel Anguilla rostrata. Comp. Biochem. Physiol., 49B; 281-290.

HOWELL, B.J.

1970 - Acid-base balance in transition from water breathing to air breathing. Fed. Proc., 1130-1134.

JOHANSEN, K.

1966 - Air breathing in the teleost Synbran. chus marmoratus. Comp. Biochem. Physiol., 18:383-395.

1970 - Air breathing in fishes. In: Fish Physiology, v. 4. New York, Academic Press.

JOHANSEN, K.; LENFANT, C.; SCHMIDT-NielseN, K. \& Petersen, J.A.

1968 - Gas exchange and control of breathing in the electric eel, Electrophorus electricus, Z. Vergl. Physiol., 61:137-163.
JOHANSEN, K.; LYKKeBoe, G.; Weber, R R, \& MALOTY, G.M.O

1976 - Respiratory properties of blood in awake and estivating lungfish, Pro. topterus amphibius. Respir. Physiol., 27:335-345.

KaLoustian, K.V. \& POLUhowich, J.J.

1976 - The role of organic hosphates in modulating the oxygenation behaviour of eel haemoglobins. Comp. Biochem. Physiol., 53A:245-248.

LyKreboe, G.; JohANSEN, K. \& MALOTy, G.M.O.

1975 - Functional properties of hemoglobins in the teleost Tilapia grahami. J. Comp. Physiol., 104:1-11.

Weber, R.E.; LYKKeboe, G. \& JoHANSEN, K.

1975 - Biochemical aspects of the adaptation of hemoglobin-oxygen affinity of eels to hypoxia. Life Sciences, 17:1345-1350

1976 - Physiological properties of eel hemoglobin: Hypoxic acclimation, phosphate effects and multiplicity. J. Exp. Biol., 64:75-88.

WEBER, R.E. \& WOOD, S.C.

1978 - Efeito de nucleosidio trifosfato eritrocitico no equilíbrio de oxigênio de hemo. globinas completa e fracionada de $\mathbf{H y}$. postomus e Pterygoplichthys "bagre" aeróbicos facultativos. Acta Amazonica $8(4)$ : Suplemento. (Este volume).

WOOD, S.C. \& JOHANSEN, K

1972 - Adaptation to hypoxia by in creased $\mathrm{HbO}_{2}$ affinity and decreased red cell ATP concentration. Nature New Biol, 237:278-279.

1973a - Organic phosphate metabolism in nucleated red cells: Influence of hypoxia on cel $\mathrm{Hb}-\mathrm{O}_{2}$ affinity. Neth. J. Sea. Res., 7:328-338.

1973b - Blood oxygen transport and acidbase balance in eels during hypoxia. Am. J. Physiol., 225:829-851.

WoOd, S.C.; Johansen, K. \& Weber, R.E.

1975 - Effects of ambient $\mathrm{PO}_{2}$ on hemoglobinoxygen affinity and red cell ATP concentrations in a benthic fish, Pleuro. nectes platessa. Respir. Physiol., 25 : 259-267.

WOOD. S.C.: WebER, R.E. \& DAVIS, B.J.

1978 - Eleitos da respiração aérea sobre o balanço ácido-base no cascudo Hypostomus sp. Acta Amazonics 8(4) : Suplemento. (Este volume). 